\title{
Electron Spin Coherence and Electron Nuclear Double Resonance of Bi Donors in Natural Si
}

\author{
Richard E. George, ${ }^{1}$ Wayne Witzel, ${ }^{2}$ H. Riemann, ${ }^{3}$ N. V. Abrosimov, ${ }^{3}$ N. Nötzel, ${ }^{3}$ \\ Mike L. W. Thewalt, ${ }^{4}$ and John J. L. Morton ${ }^{1,5, *}$ \\ ${ }^{1}$ CAESR, Clarendon Laboratory, Department of Physics, Oxford University, Oxford OX1 3PU, United Kingdom \\ ${ }^{2}$ Sandia National Laboratories, Albuquerque, New Mexico 87185, USA \\ ${ }^{3}$ Institute for Crystal Growth, Max-Born Strasse 2, D-12489 Berlin, Germany \\ ${ }^{4}$ Department of Physics, Simon Fraser University, Burnaby, British Columbia, Canada \\ ${ }^{5}$ Department of Materials, Oxford University, Oxford OX1 3PH, United Kingdom
}

(Received 23 April 2010; published 6 August 2010)

\begin{abstract}
Donors in silicon hold considerable promise for emerging quantum technologies, due to their uniquely long electron spin coherence times. Bismuth donors in silicon differ from more widely studied group V donors, such as phosphorous, in several significant respects: They have the strongest binding energy (70.98 meV), a large nuclear spin $(I=9 / 2)$, and a strong hyperfine coupling constant $(A=1475.4 \mathrm{MHz})$. These larger energy scales allow us to perform a detailed test of theoretical models describing the spectral diffusion mechanism that is known to govern the electron spin decoherence of $\mathrm{P}$ donors in natural silicon. We report the electron-nuclear double resonance spectra of the $\mathrm{Bi}$ donor, across the range $200 \mathrm{MHz}$ to $1.4 \mathrm{GHz}$, and confirm that coherence transfer is possible between electron and nuclear spin degrees of freedom at these higher frequencies.
\end{abstract}

DOI: 10.1103/PhysRevLett.105.067601

Electron and nuclear spin coherence of donors in silicon is of great importance for a number of proposals for $\mathrm{Si}$ based quantum technologies [1-4]. These schemes cite among the advantages for Si donor quantum bits: long coherence times (exceeding tens of milliseconds for the electron and seconds for the nucleus in the case of $\mathrm{Si}: \mathrm{P}$ ), high-fidelity manipulation through a combination of microwave and radio frequency pulses [5], and integration within silicon devices for measurement [6-9]. Magnetic resonance studies on $\mathrm{P}$ donors in $\mathrm{Si}$ have examined electron spin coherence in natural $\mathrm{Si}[10,11]$ and its dependence on increasing ${ }^{29} \mathrm{Si}$ concentration in the host [12], as well as the storage of coherent electron spin states in the ${ }^{31} \mathrm{P}$ nuclear spin [13].

Although there has been a focus on the $\mathrm{P}$ donor in $\mathrm{Si}$, other group $\mathrm{V}$ donors such as $\mathrm{Bi}$ also possess attractive qualities as quantum bits [14]. There have been relatively few recent studies on $\mathrm{Si} \mathrm{Bi}$, an exception being a photoluminescence study showing dynamic nuclear polarization of ${ }^{209} \mathrm{Bi}$ through optical pumping [15]. Bismuth is the deepest group $\mathrm{V}$ donor, with a binding energy of $70 \mathrm{meV}$ [16] and the largest group $\mathrm{V}$ hyperfine coupling of $1.4754 \mathrm{GHz}$ [17] to the $I=9 / 2$ nuclear spin of ${ }^{209} \mathrm{Bi}$. These parameters differ substantially from the $\mathrm{P}$ donor (44 meV and $117.52 \mathrm{MHz}$ ), raising the question of whether the same decoherence mechanisms and methodology for nuclear spin manipulation are applicable. In this Letter, we examine spin decoherence of Bi donors in natural silicon, as well as electron-nuclear double resonance (ENDOR) spectroscopy to probe the transitions of the ${ }^{209} \mathrm{Bi}$ nuclear spin. We find that these measurements compare well with $\mathrm{P}$ donors in natural silicon, providing strong motivation to pursue ${ }^{28} \mathrm{Si}: \mathrm{Bi}$ material.
PACS numbers: 76.30.Da, 03.67.-a, 71.55.Cn, 76.70.Dx

Natural Si:Bi samples were obtained from ultrapure ${ }^{\text {nat }} \mathrm{Si}$ starting material by a floating-zone technique [18] and had a room temperature resistivity of $4.5 \Omega \mathrm{cm}$ $\left(10^{16} \mathrm{Bi} / \mathrm{cm}^{-3}\right)$. Pulsed EPR and ENDOR measurements were performed by using the apparatus described in the supplementary material [19], with spectra simulated by using the EASYSPIN package [20].

The Si:Bi electron-nuclear spin system can be described by an isotropic spin Hamiltonian (in angular frequency units):

$$
\mathcal{H}_{0}=\omega_{e} S_{z}-\omega_{I} I_{z}+A \cdot \vec{S} \cdot \vec{I},
$$

where $\omega_{e}=g \beta B_{0} / \hbar$ and $\omega_{I}=g_{I} \beta_{n} B_{0} / \hbar$ are the electron and nuclear Zeeman frequencies, respectively, $g$ and $g_{I}$ are the electron and nuclear $g$ factors, respectively, $\beta$ and $\beta_{n}$ are the Bohr and nuclear magnetons, respectively, $\hbar$ is Planck's constant, and $B_{0}$ is the magnetic field applied along the $z$ axis in the laboratory frame. The donor electron spin $S=1 / 2(g=2.0003)$ is coupled to the nuclear spin $I=9 / 2$ of ${ }^{209} \mathrm{Bi}$ through a hyperfine coupling $A=$ 1475.4 MHz [17]. At high magnetic fields [i.e., $\omega_{e} \gg$ $A(I+1 / 2)]$, this leads to ten equally spaced resonances in the EPR spectrum, each corresponding to a transition $\Delta m_{S}= \pm 1$ for a given $m_{I}$ projection. As shown in Fig. 1, measurements made at the $X$ band $(9.7 \mathrm{GHz})$ are not entirely in this high-field limit. The EPR spectrum of $\mathrm{Si}$ Bi was recorded by monitoring the electron spin echo intensity as a function of magnetic field. A linewidth of $\sim 4 \mathrm{G}$ was measured for each of the ten EPR lines, consistent with inhomogeneous broadening from unresolved hyperfine coupling to the surrounding $\sim 5 \%$ abundant ${ }^{29} \mathrm{Si}$ nuclear spins. 


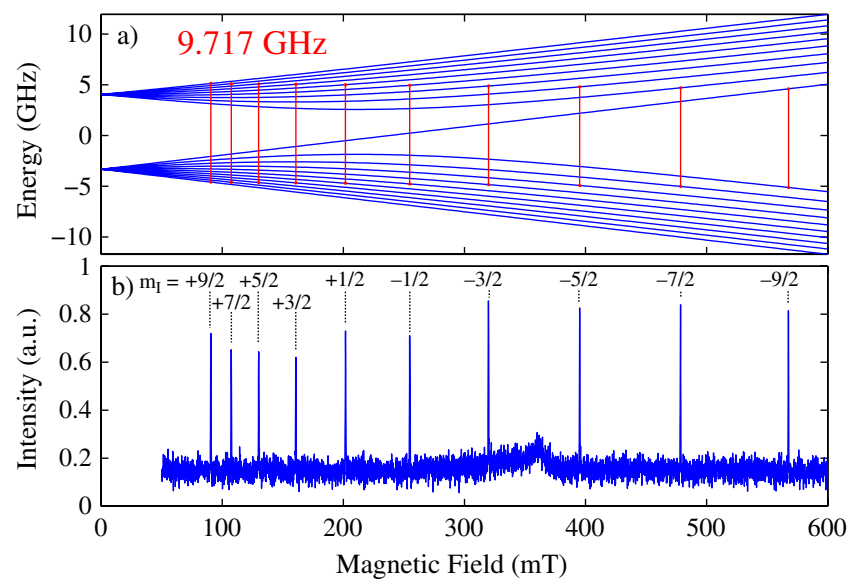

FIG. 1 (color online). Electron spin resonance of Si:Bi donors at the $X$ band $(9.7 \mathrm{GHz})$. (a) Energy levels of the coupled electron-nuclear spin system as a function of magnetic field. The allowed EPR transitions at $9.7 \mathrm{GHz}$ are indicated with vertical lines, each corresponding to a different $m_{I}$ projection for the $I=9 / 2{ }^{209} \mathrm{Bi}$ nuclear spin. (b) Experimental electron spin echo intensity as a function of magnetic field yields the 10 expected resonances. Temperature $=16 \mathrm{~K}$; simulation parameters: $A=1475.4 \mathrm{MHz}, g_{e}=2.00$, and $\gamma_{\mathrm{Bi}}=6.962 \mathrm{MHz} / \mathrm{T}$.

Electron spin decoherence of $\mathrm{P}$ donors in natural silicon is known to be dominated by spectral diffusion, a mechanism in which spin flip-flop of surrounding ${ }^{29} \mathrm{Si}$ nuclei modulates the electron Zeeman energy through both contact and dipolar hyperfine coupling [21,22]. The ${ }^{29} \mathrm{Si}$ nuclei closest to the donor are a "frozen core": They do not flipflop due to the strong spatial dependence of their coupling to the donor electron spin, causing detuning between adjacent ${ }^{29} \mathrm{Si}$ nuclear spins and suppressing nuclear flip-flop transitions that are allowed in the bulk material. Similarly, those furthest away are too weak to influence the donor electron. There is therefore an "active shell" in the region where the dipolar coupling between neighboring ${ }^{29} \mathrm{Si}$ spins is comparable to their coupling to the donor electron spin, which is responsible for spectral diffusion. This mechanism has been predicted [23,24] and shown experimentally [11] to have an angular dependence corresponding to the dependence of the dipolar coupling between nearestneighbor ${ }^{29} \mathrm{Si}$ spins on the crystal orientation with respect to the applied magnetic field. Figure 2 shows the measured electron spin echo decay traces as a function of angle, measured at the high-field line (5663 G, $\left.m_{I}=-9 / 2\right)$. The behavior is qualitatively similar to that of $\mathrm{P}$ donors in ${ }^{\text {nat }} \mathrm{Si}$, though decay times are approximately $30 \%$ longer. This can be primarily attributed to the greater binding energy of the $\mathrm{Bi}$ donor compared with $\mathrm{P}$, which shrinks the effective Bohr radius of the $\mathrm{Si}$ :Bi donor in comparison to Si:P, reducing the size of the "active region." There is also a secondary effect resulting from the much stronger hyperfine coupling to the donor nuclear spin, as described below.

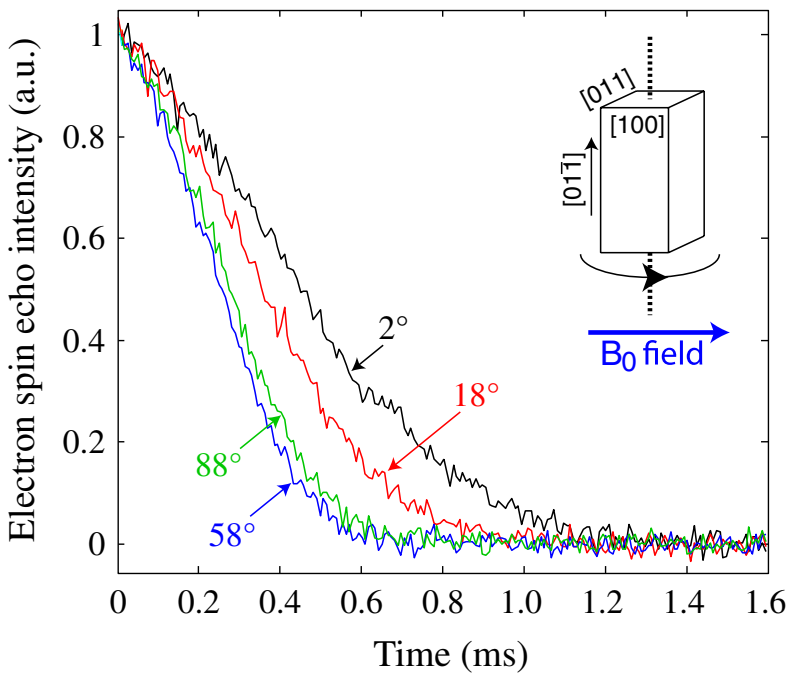

FIG. 2 (color online). Two-pulse electron spin echo decay of the $\mathrm{Si}: \mathrm{Bi}$ donor as a function of angle of the applied magnetic field $B_{0}$ with respect to the [100] crystal axis. Crystal rotation is performed in the [100]-[011] plane. $T=12 \mathrm{~K}$ and $B_{0}=$ 5663 G.

Although other group $\mathrm{V}$ donors are well into the highfield approximation at the $X$ band $(9.7 \mathrm{GHz}, 0.35 \mathrm{~T})$, the large hyperfine coupling to ${ }^{209} \mathrm{Bi}$ causes some level mixing, as shown in Fig. 1. As a measure of the sensitivity of a transition frequency $f$ to a change in magnetic field $B$, we can extract an effective gyromagnetic ratio $\gamma_{\mathrm{eff}}=d f / d B$, which differs substantially from that of a free electron $(\gamma=28.0 \mathrm{MHz} / \mathrm{T})$ and also varies for each of the hyperfine lines across the EPR spectrum: from $\gamma_{\text {eff }} \sim$ $26.7 \mathrm{MHz} / \mathrm{T}$ (for $m_{I}= \pm 9 / 2$ ) to $18.4 \mathrm{MHz} / \mathrm{T}$ (for $m_{I}=$ $\pm 1 / 2)$. The resulting change in $\gamma_{\text {eff }}$ alters the coupling of the donor electron to surrounding ${ }^{29} \mathrm{Si}$ and thus selects a different active shell, with different statistics of pairwise ${ }^{29} \mathrm{Si}$ coupling. Thus, though the dipolar coupling between neighboring ${ }^{29} \mathrm{Si}$ spins is primarily responsible for setting the time scale of spectral diffusion, we may expect an effect due to varying $\gamma_{\text {eff }}$. Through simulations based on the cluster expansion technique of Ref. [23], we have calculated the effect of varying $\gamma_{\text {eff }}$, which predicts a $\sim 5 \%$ increase in spectral diffusion times $T_{\mathrm{SD}}$ as measured on the $m_{I}= \pm 1 / 2$ hyperfine line, compared to $m_{I}=$ $\pm 9 / 2$. The simulations use a Kohn-Luttinger wave function with the $70 \mathrm{meV}$ binding energy for $\mathrm{Bi}$.

We fit the echo decay traces, such as those in Fig. 2, to a combination of an orientation-independent $T_{2}$, combined with an orientation-dependent $T_{\mathrm{SD}}$, through an expression of the form:

$$
V(t)=V_{0} e^{-\left(t / T_{2}\right)-\left(t / T_{\mathrm{SD}}\right)^{n}} .
$$

The spectral diffusion mechanism has a characteristic stretched exponential coherence decay, with a typical value of $n$ between 1 and 4 depending on the regime of spectral diffusion [23,25-27]. The stretching factor $n$ was found to 
be independent of crystal orientation, while $T_{\mathrm{SD}}$, plotted in Fig. 3, shows the expected orientation dependence with a maximum when the applied magnetic field $B_{0}$ is oriented along [100] and a minimum when oriented along [111]. Comparing the values measured at two hyperfine lines, $m_{I}=-1 / 2(2542 \mathrm{G})$ and $m_{I}=-9 / 2(5663 \mathrm{G})$, we see agreement with the $\gamma_{\text {eff }}$ dependence predicted by the simulations. The stretching factor $n$ showed no significant field dependence: $n_{2542 \mathrm{G}}=2.30(7)$ and $n_{5663 \mathrm{G}}=2.34(5)$, consistent with the simulations which predict 2.30(1).

The inset in Fig. 3 shows the predicted values of $T_{\mathrm{SD}}$ from the simulations, which are within $\sim 20 \%$ of the experimental values. The magnetic field dependence of $T_{\mathrm{SD}}$ is also well represented, showing a maximum for $m_{I}=$ $\pm 1 / 2$. The simulations were performed by using the cluster correlation expansion [28], but it is sufficient to use a simple pair approximation that includes effects from each pair of nuclear spins independently [26,29].

The fitting error in the residual $T_{2}$ parameter is large when it is much longer than $T_{\mathrm{SD}}$; however, our extracted values of a few milliseconds are consistent with being limited by electron spin relaxation $\left(T_{1}\right)$ at this temperature (12 K). We find that $T_{1}$ is well described by a first-order Raman mechanism $\left(T^{-7}\right)$ in the temperature range $8-16 \mathrm{~K}$, as proposed by Castner for the range 19-25 K [30] (see supplementary material [19]).

To study the ${ }^{209} \mathrm{Bi}$ nuclear spin, we used the Davies ENDOR sequence $\left(\pi_{\mathrm{mw}}-\pi_{\mathrm{rf}}-\pi / 2_{\mathrm{mw}}-\tau-\pi_{\mathrm{mw}}-\right.$

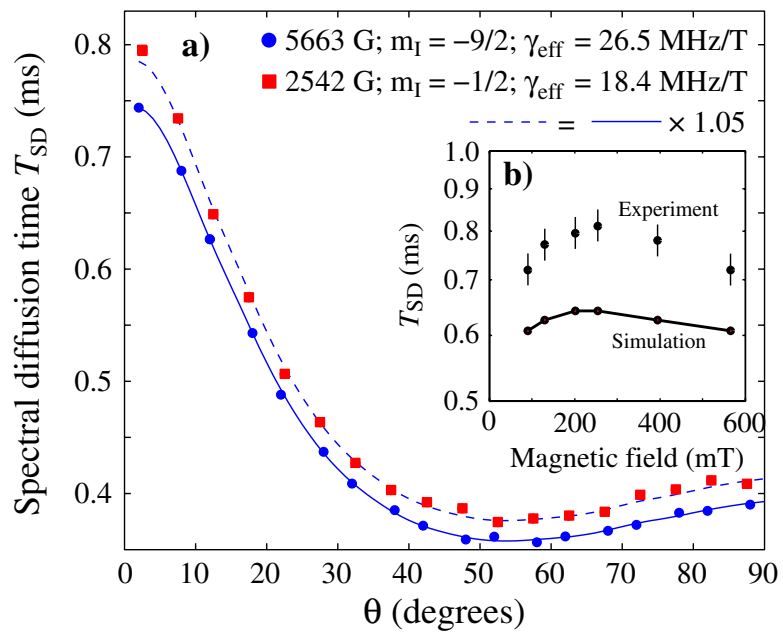

FIG. 3 (color online). Extracted spectral diffusion times $T_{\mathrm{SD}}$ as a function of crystal orientation and magnetic field. (a) A fit to the electron spin echo decay curves provides a measure of $T_{\mathrm{SD}}$ as a function of the angle of the applied magnetic field $B_{0}$ with respect to the [100] crystal axis, performed on two of the ten hyperfine lines: 5663 (blue circles) and $2542 \mathrm{G}$ (red squares). The solid curve is a spline fit through the data for $5663 \mathrm{G}$, which is multiplied by 1.05 to produce the dashed curve. (b) The inset shows the magnetic field dependence of $T_{\mathrm{SD}}$ for six field positions $\left(\theta=0^{\circ}\right)$, showing good agreement with the results of the simulation (see text). Temperature $=12 \mathrm{~K}$. $\tau$ - echo) at each of the ten hyperfine lines to map out the set of 36 distinct ENDOR lines observable at a given microwave frequency: For each hyperfine line (i.e., EPR transition) there are four observable ENDOR transitions $\left(\Delta m_{I}=1\right)$, apart from at the ends of the EPR spectrum $\left(m_{I}= \pm 9 / 2\right)$ where there are only two. The measured frequencies are shown in Fig. 4(a), along with theoretical curves showing ENDOR frequencies as a function of magnetic field.

Two typical ${ }^{209} \mathrm{Bi}$ ENDOR lines are shown in Figs. 4(b) and 4(c). The ENDOR linewidth ranges from 100 to $700 \mathrm{kHz}$, depending on the particular transition and field being measured. In general, the linewidths are broader at lower magnetic fields or $\mathrm{rf}$ frequencies, except for the two lines corresponding to the $m_{I}=-9 / 2:-7 / 2$ transition which are about $350 \mathrm{kHz}$ wide. The ENDOR linewidths we observe (plotted in the supplementary material [19]) are well described by a combination of two factors: At lower magnetic fields $(<400 \mathrm{mT})$, the linewidth arises from the random dipolar field of surrounding ${ }^{29} \mathrm{Si}$, as in the case for the EPR linewidth. This effect is directly related to the gradient of the field versus frequency curves shown in Fig. 4(a), which flatten out as the high-field approximation becomes valid (this also accounts for the greater linewidth of the $m_{I}=-9 / 2:-7 / 2$ transition). Correspondingly, this broadening mechanism is not significant for ENDOR in other group $\mathrm{V}$ donors at the $X$ band given their much weaker hyperfine couplings. Instead, ENDOR linewidth in such donors arises from an inhomogeneity in the hyperfine coupling to the donor nucleus, due to a variation in the dielectric constant of the material within the donor wave function caused by the random distribution of ${ }^{29} \mathrm{Si}$. Such a mechanism could be responsible for the ENDOR linewidth in Si:Bi at higher magnetic field $(<400 \mathrm{mT})$, and from our measurements we can put an upper bound of the inhomogeneity in $a\left({ }^{209} \mathrm{Bi}\right)$ to be $<0.02 \%$.
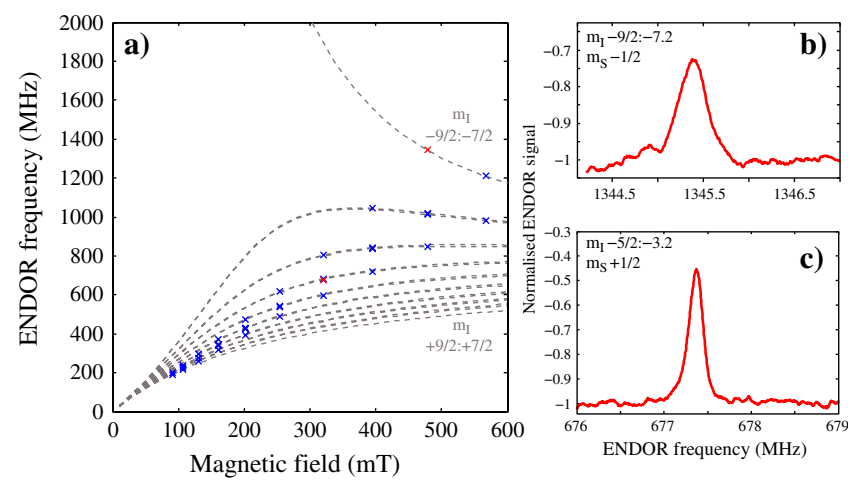

FIG. 4 (color online). ENDOR of ${ }^{209} \mathrm{Bi}$ in silicon. (a) Dashed curves show the theoretical ENDOR frequencies as a function of field, for each $\Delta m_{I}=1$ transition. Symbols represent those values measured by Davies ENDOR at the $X$ band, at each of the 10 resonant fields of the EPR spectrum. (b) and (c) show two typical ENDOR peaks, at 1345.4 and $677.4 \mathrm{MHz}$ with rf $\pi$-pulse length $=4$ and $7 \mu \mathrm{s}$, respectively. 
It has been shown that the ${ }^{31} \mathrm{P}$ donor nuclear spin can provide a valuable resource for storing the coherent state of the electron spin for times exceeding seconds [13]. The larger nuclear spin $(I=9 / 2)$ of ${ }^{209} \mathrm{Bi}$ provides a correspondingly larger Hilbert space for storing electron spin qubits, though it also introduces more potential relaxation mechanisms for the nuclear spin. As we have found for $\mathrm{Si}: \mathrm{P}$, high-fidelity storage or retrieval of the electron coherence requires narrow EPR and ENDOR lines and thus a ${ }^{28} \mathrm{Si}$-enriched host material. We have investigated the potential of the ${ }^{209} \mathrm{Bi}$ nuclear spin for quantum memory and found that, as expected, the ${ }^{\text {nat }} \mathrm{Si}$ host limits the store and retrieve fidelity to $\sim 63 \%$ (see supplementary material [19]). We can nevertheless measure the decay of nuclear coherence and found that it is limited by the effect of $T_{1 e}$ (first-order Raman) processes at temperatures above $10 \mathrm{~K}$-random fluctuations in the electron polarization drive decoherence of the strongly coupled nuclear spin. Below this temperature, $T_{2 n}$ is limited to about $15 \mathrm{~ms}$. Further work using ${ }^{28} \mathrm{Si}: \mathrm{Bi}$ will be required to explore this limit and investigate the sources of nuclear decoherence.

We have found electron spin decoherence of Bi donors in natural Si to be dominated by the same spectral diffusion mechanism found in the case of Si:P; however, thanks to the smaller Bohr radius of $\mathrm{Bi}$, the effect is weaker than for $\mathrm{P}$, leading to $30 \%$ longer $T_{2}$ times. Despite the high ENDOR frequencies necessary to probe the ${ }^{209} \mathrm{Bi}$ nuclear transitions, it is possible to excite each transition with a fidelity determined by the ENDOR linewidth of a few $100 \mathrm{kHz}$, allowing us to demonstrate the possibility of storage and retrieval of electron spin coherence in the ${ }^{209} \mathrm{Bi}$ nuclear spin. Applying such techniques to ${ }^{28} \mathrm{Si}: \mathrm{Bi}$, we would anticipate the ability to store and retrieve multiple electron spin qubits with high fidelity within the nuclear spin. Finally, we note that the large energy splitting present at zero applied magnetic field makes the Si:Bi donor spin an attractive candidate for coupling to superconducting resonators.

We thank Alexei Tyryshkin, Steve Lyon, Arzhang Ardavan, and Andrew Briggs for helpful discussions. J. J.L.M. is supported by the Royal Society. The research is supported by the EPSRC through CAESR (No. EP/ D048559/1). Sandia National Laboratories is a multiprogram laboratory operated by Sandia Corporation, a wholly owned subsidiary of Lockheed Martin company, for the U.S. Department of Energy's National Nuclear Security Administration under Contract No. DE-AC04-94AL85000.

*john.morton@materials.ox.ac.uk

[1] B. E. Kane, Nature (London) 393, 133 (1998).

[2] A. J. Skinner, M. E. Davenport, and B. E. Kane, Phys. Rev. Lett. 90, 087901 (2003).
[3] R. Vrijen, E. Yablonovitch, K. Wang, H. W. Jiang, A. Balandin, V. Roychowdhury, T. Mor, and D. DiVincenzo, Phys. Rev. A 62, 012306 (2000).

[4] J. J. L. Morton, arXiv:0905.4008.

[5] J. J. L. Morton, A. M. Tyryshkin, A. Ardavan, K. Porfyrakis, S. A. Lyon, and G. A. D. Briggs, Phys. Rev. Lett. 95, 200501 (2005).

[6] A. Morello, C. Escott, H. Huebl, H. WillemsvanBeveren, L. Hollenberg, D. Jamieson, A. Dzurak, and R. Clark, Phys. Rev. B 80, 081307(R) (2009).

[7] K. Y. Tan et al., Nano Lett. 10, 11 (2010).

[8] F. Hoehne, H. Huebl, B. Galler, M. Stutzmann, and M. S. Brandt, Phys. Rev. Lett. 104, 046402 (2010).

[9] C. C. Lo, J. Bokor, T. Schenkel, A. M. Tyryshkin, and S. A. Lyon, Appl. Phys. Lett. 91, 242106 (2007).

[10] A. M. Tyryshkin, S. A. Lyon, A. V. Astashkin, and A. M. Raitsimring, Phys. Rev. B 68, 193207 (2003).

[11] A. M. Tyryshkin, J.M.S. Benjamin, A. Ardavan, G. Briggs, J.W. Ager, and S. A. Lyon, J. Phys. Condens. Matter 18, S783 (2006).

[12] E. Abe, K. M. Itoh, J. Isoya, and S. Yamasaki, Phys. Rev. B 70, 033204 (2004).

[13] J. J. L. Morton, A. M. Tyryshkin, R. M. Brown, S. Shankar, B. W. Lovett, A. Ardavan, T. Schenkel, E. E. Haller, J.W. Ager, and S. Lyon, Nature (London) 455, 1085 (2008).

[14] A. Stoneham, A. Fisher, and P. Greenland, J. Phys. Condens. Matter 15, L447 (2003).

[15] T. Sekiguchi, M. Steger, K. Saeedi, M. L. W. Thewalt, H. Riemann, N. V. Abrosimov, and N. Nötzel, arXiv:0912.3849.

[16] N. R. Butler, P. Fisher, and A. K. Ramdas, Phys. Rev. B 12, 3200 (1975).

[17] G. Feher, Phys. Rev. 114, 1219 (1959).

[18] H. Riemann, N. Abrosimov, and N. Nötzel, ECS Trans. 3, 53 (2006).

[19] See supplementary material at http://link.aps.org/ supplemental/10.1103/PhysRevLett.105.067601 for supporting information on electron spin relaxation, ENDOR, and nuclear memory experiments.

[20] S. Stoll and A. Schweiger, J. Magn. Reson. 178, 42 (2006).

[21] J. R. Klauder and P. W. Anderson, Phys. Rev. 125, 912 (1962).

[22] K. M. Salikhov, S.A. Dzuba, and A.M. Raitsimring, J. Magn. Reson. 42, 255 (1981).

[23] W. M. Witzel and S. Das Sarma, Phys. Rev. B 74, 035322 (2006).

[24] R. de Sousa and S. Das Sarma, Phys. Rev. B 68, 115322 (2003).

[25] A.D. Milov, K. M. Salikhov, and Y.D. Tsvetkov, Fiz. Tverd. Tela (Leningrad) 15, 1187 (1973).

[26] W. Yao, R.-B. Liu, and L. J. Sham, Phys. Rev. B 74, 195301 (2006).

[27] W. A. Coish, J. Fischer, and D. Loss, Phys. Rev. B 77, 125329 (2008).

[28] W. Yang and R.-B. Liu, Phys. Rev. B 78, 085315 (2008).

[29] W. M. Witzel, R. de Sousa, and S. Das Sarma, Phys. Rev. B 72, 161306(R) (2005).

[30] T. G. Castner, Phys. Rev. 130, 58 (1963). 